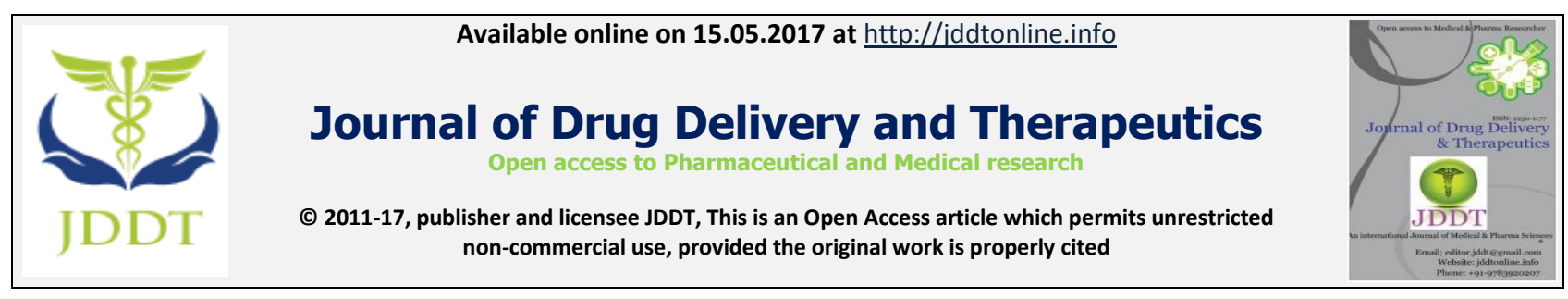

Mini Review

\title{
Plga Nanomedicine
}

\section{Xiaochai Zhu}

School of Pharmacy, Nanjing University of Chinese Medicine, Nanjing, Jiangsu, PR China, 210023

Article Info: Received 25 April 2017; $\quad$ Review Completed 07 May 2017; $\quad$ Accepted 07 May 2017, Available online 15 May 2017

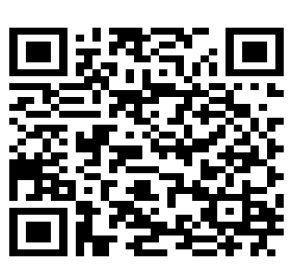
Cite this article as:

Zhu X, PLGA Nanomedicine, Journal of Drug Delivery and Therapeutics. 2017; 7(3):84-86

DOI: http://dx.doi.org/10.22270/jddt.v7i3.1452

*Address for Correspondence:

Xiaochai Zhu, School of Pharmacy, Nanjing University of Chinese Medicine, Nanjing, Jiangsu, PR China, 210023. Email: zhuxiaochai@126.com

\section{ABSTRACT}

Poly lactic-co-glycolic acid (PLGA) based nanoparticles are wildly used for the delivery of bioactive compounds such as dexamethasone, curcumin, azithromycin, doxorubicin and indocyanine green. It was found that those polymeric compounds can be effectively used for nanoparticle drug delivery. They are more biocompatible and lesser resistant in the case of targeted drug delivery. These are observed to have wide-ranging applications. Many of the PLGA polymers are being currently under research for commercial use and treatment. They have many fabrication techniques. It is imperative to understand their limitations in the future. There is a need for more research to overcome these limitations to develop drugs that are more efficient.

Keywords: PLGA, Dexamethasone, Curcumin, nanomedicine, Indocyanine green.

\section{INTRODUCTION}

Poly lactic-co-glycolic acid (PLGA) is some of the important polymeric compounds that for the fabricate devices for the drug delivery. ${ }^{1}$ They have been garnering attention in the recent times. It also has application in tissue engineering application. The PLGA is a compound that is biocompatible and biodegradable. This compound has many applications and finds its uses in many fields. ${ }^{2-4}$ PLGA is an FDA-approved polymer. In particular, PLGA is studied for the development of devices that is relevant for the small molecule drugs and other macromolecules. ${ }^{5}$ This is observed to have used in commercial purposes and research. ${ }^{6,7}$ Many fabrication techniques are applied in the devices. ${ }^{8-10}$ These are utilizing to understand the factors that affect the degradation and drug release of compound. ${ }^{11-13}$ Many of these compounds are useful in many operational factors. ${ }^{14-17}$ Some of them are detailed in this analysis. In the subsequent section, there will be the discussion of some of the different kinds of PLGA that is commercially used for the treatment of the diseases.

Cancer - Doxorubicin
Anthracyclines is observed intercalate with the DNA in vitro. This is an essential functionality of the compound that finds its application in many sectors. It is observed to encompass several crystal structures complexes of the DNA. In this case, the Doxorubicin (DOX) breaks the DNA and interferes with the DNA synthesis. This particular function is imperative for the treatment of the compounds. This is the main reason for the compound to have pharmacological uses. It is observed that the DNA-DOX interaction subsequently leads to the poisoning of the topoisomerase II (TOP2A). There is a translocation of the DOX into the nucleus and is thought to bind with the proteasomes. The TOP2A poisonmediated cytotoxicity involves itself with the mismatching of the repair genes MSH2 and MLH1. There is a loss of DNA owing to the mismatch repair functions. This results in the resistance of the doxorubicin. ${ }^{18}$ Topoisomerase II-DNA is damaged in the process, and this ensures the cell death. TP53, a gene, is the gene that plays a major role in the apoptosis and DNA-damage response. This increases the up-regulation of TP53 and is found to impact the anthracycline treatment. The ERCC2 and TP53 interact with the p53mediated apoptotic pathway with the DOX treatment. 
The DOX undergoes one-electron reduction and forms a DOX-semiquinone radical. These enzymes include the mitochondrial NADH dehydrogenase that is present in the sarcoplasmic reticulum along with the mitochondria. One of the main limitations for this therapeutic compound is the toxicity and resistance of the compounds. The whole potential of the complex is yet to analyze completely.

\section{Tumor Imaging -- Indocyanine green (ICG)}

Indocyanine green functions as a tricarbocyanine dye that is used for diagnose liver function and determine the cardiac output and the blood volume of the compounds. This ICG compound finds its uses in the sentinel lymph node biopsy. ${ }^{19-22}$ It allows the selective and minimally invasive access to the regional lymph node status along with the malignant tumors. The first drain lymph node is known as the "sentinel." This identifies the tumor in the lymph node region. The method is then confirmed with the radionuclides. The ICG compounds are exploited as it enables in the high rate of detection and sensitivity of the compounds in the conventional methods. There is a need to achieve high rates of deduction and sensitivity in comparison with the traditional methods. There is some in vivo experiments that showcase the FA-ICG-PLGA-lipid NPs. ${ }^{23}$ They are biocompatible and biodegradable in NIR-NPs. These have potential uses in the tumor imaging based on the high aqueous stability and its fundamental NIR optical properties. These are the reasons for considerations. It is expected that in the future there will be an increase in the tumor imaging process based on the ICG methods.

\section{Azithromycin - antibacterial}

Azithromycin-loaded poly (lactide-co-glycolide) (PLGA) nanoparticles have anti-bacterial properties. They impact of the anti-bacterial impacts varied based on the drug to polymer ratio. There is also high entrapment efficiency. The nanoparticles are prepared by nanoprecipitation technique. ${ }^{24}$ The antimicrobial activity shows that the nanoparticles were more effective than the azithromycin. Clinical activity of the azithromycin can be achieved by enhancing nanoparticle formulation of the drug. This increases the effective therapy when compared to the conventional formulation of the azithromycin. There are some clinical trials that are being undertaken in the current times to address the antibacterial functions of the medicines.

\section{Curcumin -- Alzheimer's disease}

Curcumin has anti-inflammatory properties. It is extracted from the roots of the turmeric plant. These have been used in conventional medications for many centuries. $^{25-27}$ It is a yellow pigment, and it has a polyphenolic molecular structure. ${ }^{28-30}$ Curcumin compounds have the potential for the prevention and progression of Alzheimer's. ${ }^{31}$ This is a multi-functional drug. When Tet-1 targeted PLGA was coated with curcumin nanoparticles, it observed treat AD with antiamyloid property and anti-oxidant property. By tagging of the PLGA- curcumin nanoparticle with the Tet-1 neuropeptide, there was an increase in the efficiency of the processes. The preliminary study indicates that there is a potential role for Tet-1 neuropeptide in the treatment of Alzheimer's. The impact of the Tet-1 peptide is in the neuronal targeting of the Nanoparticles yield positive results. Added to this, curcumin has been observed to have a biocompatible nature of the curcumin. Owing to these factors, this PLGA compound is being studied. Apart from this, the curcumin has some positive potential impacts in other body conditions. It is because of these factors that this compound is being extensively studied.

\section{Dexamethasone -- bone disease}

Dexamethasone is a corticosteroid. It is a natural hormone and is utilized for the production of the adrenal glands. ${ }^{32-34}$ It is used to replace the chemicals when the body does not make enough of such compounds. It is also utilized for the alleviation of inflammation, cancer treatment and has used in in palliative care. ${ }^{35-37}$ These oppositely- charged Poly (D, L-lactic-co-glycolic acid) (PLGA) is converted into a colloidal gel and is filled with an injectable drug-loaded filler to promote the bond defects. ${ }^{38}$ These colloids have been observed to have self-assembly and stable 3-D networks. These are then molded or extruded based on the share. Once the external force was removed, they have a cohesive property of the colloidal gel. These are observed to have reversibility and shear-thinning behavior. Zero-order dexamethasone release was observed in two months. These drugs were then encapsulated in PLGA nanoparticles, and there was a blending of the drug with the colloidal gel. There was a pseudoplastic material which showcases that this PLGA colloidal Gel gets stimulated and plays an important role in osteoconductive bone formation in the cranial bone defects of the rat. From this, it can be inferred that pseudoplastic, shear-thinning behavior of the colloidal gets are expedited for the application of the injectable scaffold. This has their uses for the handling of the tissue damage and repair. The full potential of the Dexamethasone as a PLGA colloidal gel is being studied in in-vivo and is yet to be completely analyzed. The results have so far been promising.

\section{SUMMARY}

Poly lactic-co-glycolic acid (PLGA) compounds such as Dexamethasone, Curcumin, Azithromycin-loaded poly (lactide-co-glycolide) (PLGA) nanoparticles; Doxorubicin and Indocyanine Green was analyzed in detail in this analysis. It has been determined that these polymeric compounds are used for Nanoparticle specific drug delivery. These have wide-ranging applications and are biocompatible. They are an FDA-approved polymer. PLGA compound is utilized for the development of devices for the small molecule drugs and in other macromolecules. These have applications in commercial uses and research. They have many fabrication techniques. It is imperative to understand these factors that impact the degradation and drug release of the compounds. Each one of the elements that has been discussed in this analysis has specific uses. The complete commercial use and analysis are yet to be done on a larger scale. More research needs to be in in-vivo to determine the efficacy of these PLGA compounds. 


\section{REFERENCES}

1. Park J H, Lee S, Kim J-H, Park K, Kim K, and Kwon I C. Polymeric nanomedicine for cancer therapy. Progress in Polymer Science. 2008, 33: 113-137

2. Nishiyama N. Nanomedicine: nanocarriers shape up for long life. Nature Nanotechnology. 2007, 2: 203

3. Duan Y, Pei K, Cai H, Tu S, Zhang Z, Cheng X, et al. Bioactivity evaluation-based ultra high-performance liquid chromatography coupled with electrospray ionization tandem quadrupole-time-offlight mass spectrometry and novel distinction of multisubchemome compatibility recognition strategy with Astragali Radix-Fructus Corni herb-pair as a case study. Journal of pharmaceutical and biomedical analysis. 2016, 129: 514-534

4. Cheng X. Developing organic and inorganic nanomedicine for cancer therapy. Journal of Drug Delivery and Therapeutics. 2017; 7(3):1-4. DOI: 10.22270/jddt.v7i2.1367

5. Kumari A, Yadav S K, and Yadav S C. Biodegradable polymeric nanoparticles based drug delivery systems. Colloids and Surfaces B: Biointerfaces. 2010, 75: 1-18

6. Li H, Cheng X, Liu Y, Lee Y B, Kim D J, Ahn C-h, et al. Folate receptor-targeted lipid coated albumin nanoparticles (F-LCAN) for therapeutic delivery of RX-0201 (Archexin $\left.{ }^{\circledR}\right)$, an antisense oligonucleotide against Akt-1. 2016,

7. Cheng X, Liu Q, Li H, Kang C, Liu Y, Guo T, et al. Lipid Nanoparticles Loaded with an Antisense Oligonucleotide Gapmer Against Bcl-2 for Treatment of Lung Cancer. Pharm Res. 2017, 34: 310-320; doi: 10.1007/s11095-016-2063-5.

8. Sun Y, Kang C, Liu F, and Song L. Delivery of Antipsychotics with Nanoparticles. Drug Development Research. 2016, 77: 393-399

9. Kang C, Sun Y, Zhu J, Li W, Zhang A, Kuang T, et al. Delivery of Nanoparticles for Treatment of Brain Tumor. Current Drug Metabolism. 2016, 17: 745-754

10. Xue X, Zhao N-Y, Yu H-T, Sun Y, Kang C, Huang Q-B, et al Discovery of novel inhibitors disrupting HIF-1 $\alpha$ /von Hippel-Lindau interaction through shape-based screening and cascade docking. PeerJ. 2016, 4: e2757

11. Li H, Quan J, Zhang M, Yung B C, Cheng X, Liu Y, et al. LipidAlbumin Nanoparticles (LAN) for Therapeutic Delivery of Antisense Oligonucleotide against HIF-1alpha. Mol Pharm. 2016, 13: 2555-2562; doi: 10.1021/acs.molpharmaceut.6b00363.

12. Cheng $X$, and Lee $R$ J. The role of helper lipids in lipid nanoparticles (LNPs) designed for oligonucleotide delivery. $A d v$ Drug Deliv Rev. 2016, 99: 129-137; doi: 10.1016/j.addr.2016.01.022.

13. Duan Y, Pei K, Cai H, Tu S, Cheng X, Zhang Z, et al. Strategy of integrated evaluation on treatment of traditional Chinese medicine as 'interaction of system to system' and establishment of novel fuzzy target contribution recognition with herb-pairs, a case study on Astragali Radix-Fructus Corni. Molecular and Cellular Endocrinology. 2016, 434: 219-237

14. Campolongo M J, Tan S J, Xu J, and Luo D. DNA nanomedicine: Engineering DNA as a polymer for therapeutic and diagnostic applications. Advanced drug delivery reviews. 2010, 62: 606-616

15. Han R, Sun Y, Kang C, Sun H, and Wei W. Amphiphilic dendritic nanomicelle-mediated co-delivery of 5-fluorouracil and doxorubicin for enhanced therapeutic efficacy. Journal of Drug Targeting. 2017 , 25: $140-148$

16. Sun Y, Kang C, Zhang A, Liu F, Hu J, Zhong X, et al. Co-delivery of dual-drugs with nanoparticle to overcome multidrug resistance. European Journal of BioMedical Research. 2016, 2: 12-18

17. Song L, Kang C, Sun Y, Huang W, Liu W, and Qian Z. Crocetin Inhibits Lipopolysaccharide-Induced Inflammatory Response in Human Umbilical Vein Endothelial Cells. Cellular Physiology and Biochemistry. 2016, 40: 443-452

18. Park J, Fong P M, Lu J, Russell K S, Booth C J, Saltzman W M, et al. PEGylated PLGA nanoparticles for the improved delivery of doxorubicin. Nanomedicine: Nanotechnology, Biology and Medicine. 2009, 5: 410-418

19. Liu Z-1, Zhang R-m, Meng Q-g, Zhang X-c, and Sun Y. Discovery of new protein kinase CK2 inhibitors with 1, 3-dioxo-2, 3-dihydro-1 H-indene core. MedChemComm. 2016, 7: 1352-1355
20. Sun H, Zhu J, Chen Y, Sun Y, Zhi H, Li H, et al. Docking Study and Three-Dimensional Quantitative Structure-Activity Relationship (3D-QSAR) Analyses and Novel Molecular Design of a Series of 4-Aminoquinazolines as Inhibitors of Aurora B Kinase. Chinese Journal of Chemistry. 2011, 29: 1785-1799

21. Guo X K, Sun H P, Shen S, Sun Y, Xie F L, Tao L, et al. Synthesis and evaluation of gambogic acid derivatives as antitumor agents. Part III. Chemistry \& biodiversity. 2013, 10: 73-85

22. Zhou Y, Xu X, Sun Y, Wang H, Sun H, and You Q. Synthesis, cytotoxicity and topoisomerase II inhibitory activity of lomefloxacin derivatives. Bioorganic \& medicinal chemistry letters. 2013, 23: 2974-2978

23. Zheng $\mathrm{C}$, Zheng $\mathrm{M}$, Gong $\mathrm{P}$, Jia D, Zhang $\mathrm{P}$, Shi $\mathrm{B}$, et al. Indocyanine green-loaded biodegradable tumor targeting nanoprobes for in vitro and in vivo imaging. Biomaterials. 2012, 33: 5603-5609

24. Mohammadi G, Valizadeh H, Barzegar-Jalali M, Lotfipour F, Adibkia K, Milani M, et al. Development of azithromycin-PLGA nanoparticles: Physicochemical characterization and antibacterial effect against Salmonella typhi. Colloids and Surfaces B: Biointerfaces. 2010, 80: 34-39

25. Chen Y, Bian Y, Sun Y, Kang C, Yu S, Fu T, et al. Identification of 4-aminoquinoline core for the design of new cholinesterase inhibitors. PeerJ. 2016, 4: e2140

26. Kang C, Sun Y, Wang M, and Cheng X. Nanosized camptothecin conjugates for single and combined drug delivery. European Journal of BioMedical Research. 2016, 2: 8-14

27. Qiao H, Fang D, Chen J, Sun Y, Kang C, Di L, et al. Orally delivered polycurcumin responsive to bacterial reduction for targeted therapy of inflammatory bowel disease. Drug Delivery. 2017, 24: 233-242

28. XU S-h, Chen K, CHEN M-1, ZHOU P-p, HE G-w, CUI Y-j, et al. Dynamic expression of AQP4 in early stageof ischemia/reperfusion rats and cerebral edema. Chinese Pharmacological Bulletin. 2016: 1433-1441

29. Yang Z, Xie J, Zhu J, Kang C, Chiang C, Wang X, et al. Functional exosome-mimic for delivery of siRNA to cancer: in vitro and in vivo evaluation. Journal of Controlled Release. 2016, 243: 160-171

30. Waller A P, George M, Kalyanasundaram A, Kang C, Periasamy M, $\mathrm{Hu} \mathrm{K}$, et al. GLUT12 functions as a basal and insulin-independent glucose transporter in the heart. Biochimica et Biophysica Acta (BBA)-Molecular Basis of Disease. 2013, 1832: 121-127

31. Mathew A, Fukuda T, Nagaoka Y, Hasumura T, Morimoto H, Yoshida $\mathrm{Y}$, et al. Curcumin loaded-PLGA nanoparticles conjugated with Tet-1 peptide for potential use in Alzheimer's disease. PLoS one. 2012, 7: e32616

32. Liu F, Sun Y, Kang C, and Zhu H. Pegylated Drug Delivery Systems: From Design to Biomedical Applications. Nano LIFE. 2016, 6: 1642002

33. Sun Y, Kang C, Yao Z, Liu F, and Zhou Y. Peptide-Based Ligand for Active Delivery of Liposomal Doxorubicin. Nano LIFE. 2016, 6: 1642004

34. Kang C, Qin J, Osei W, and Hu K. Regulation of protein kinase Cepsilon and its age-dependence. Biochemical and Biophysical Research Communications. 2017, 482: 1201-1206

35. Sun Y, and Kang C. Self-Assembly of Peptides into Hydrogel. Journal of Organic \& Inorganic Chemistry. 2016,

36. Yao Z, Sun Y, and Kang C. Structure and Self-Assembly of Multicolored Naphthalene Diimides Semiconductor. Nano LIFE. 2016, 6: 1642007

37. Zhong X, Sun Y, Kang C, and Wan G. The theory of dielectrophoresis and its applications on medical and materials research. European Journal of BioMedical Research. 2017, 2: 7-11

38. Wang Q, Wang J, Lu Q, Detamore M S, and Berkland C. Injectable PLGA based colloidal gels for zero-order dexamethasone release in cranial defects. Biomaterials. 2010, 31: $4980-4986$ 